\title{
Lake Baikal Endemic Sculpins (Cottoidei): A Promising Model to Study Adaptive Plasticity of Blood Cholesterol Metabolism
}

\author{
Nikolay P. Sudakov ${ }^{1,2,3^{*}}$, Igor V. Klimenkov ${ }^{2,4}$ and Mikhail V. Pastukhov ${ }^{5}$ \\ ${ }^{I}$ Scientific Center for Reconstructive and Restorative Surgery; Siberian Branch, Russian Academy of Medical \\ Sciences; Irkutsk - Russia. ${ }^{2}$ Irkutsk State University; Irkutsk - Russia. ${ }^{3}$ Irkutsk Scientific Center, Siberian Branch, \\ Russian Academy of Sciences; Irkutsk - Russia. ${ }^{4}$ Limnological Institute, Siberian Branch; Russian Academy of \\ Sciences; Irkutsk - Russia. ${ }^{5}$ Vinogradov Institute of Geochemistry, Siberian Branch; Russian Academy of Sciences; \\ Irkutsk - Russia
}

\begin{abstract}
We analyzed the blood lipid spectra in four closely related sculpin (Cottoidei) species endemic to Lake Baikal. These data characterize the Baikal sculpins as a set of model organisms for studying the adaptive plasticity of cholesterol metabolism and also mechanisms of resistance to the development of dyslipidemia and atherosclerosis.
\end{abstract}

Key words: cholesterol, lipoproteins, liver, Cottoidei, Lake Baikal

\section{INTRODUCTION}

Blood lipid metabolism in different animals has certain specific features that determine the degree of their resistance to the development of dyslipidemia and atherosclerosis. Analysis of such species-specific differences offers an opportunity to gain an insight into the mechanisms of this resistance and, on this basis, to develop new approaches to the prevention and treatment of atherosclerosis in humans. Such studies are being performed on animal models from different taxonomic groups, such as mammals, birds, and fishes (Getz and Reardon 2012). However, these groups are phylogenetically distant from each other, which make it difficult to adequately compare biochemical data in order to reveal general mechanisms underlying the adaptive plasticity of cholesterol metabolism. To solve this important problem, it appears more expedient to analyze closely related organisms that occupy different ecological niches and, hence, differ in parameters of lipid metabolism. In search for such models, we have focused attention on the group of closely related sculpins (Cottoidei) endemic to Lake Baikal. This group consists of 26 species that have originated from a few ancestral forms (Sideleva 2003) but are highly diverse, being adapted to different ambient conditions and diets that present different levels of atherogenic risk. It is important that anthropogenic pollution of Lake Baikal is minimal (Shimaraev et al. 2000) and, hence, cannot cause artifacts in studies on blood cholesterol metabolism as related to adaptive radiation in the Cottoidei. Moreover, the liver in fish plays a major role in lipid storage (Henderson and Torcher 1987), but large-scale accumulation of lipid droplets in liver cells does not lead to dyslipidemia and atherosclerosis, which inevitably develop under such conditions in mammals,

*Author for correspondence: npsudakov@ rambler.ru 
including humans. Thus, fish models are promising for the study of mechanisms responsible for resistance to steatosis, steatohepatitis, and disturbances of blood lipid metabolism.

The study was performed with representatives of four Cottoidei species caught in the coastal zone of southern Lake Baikal: Procottus major, Taliev, $1944(n=10) ;$ Cottocomephorus inermis, Yakovlev, $1890(n=10)$, Limnocottus bergianus, Taliev, $1935(n=10)$; and Batrachocottus baicalensis, Dybowski, $1874(n=10)$. Data on their size, weight, sex, and age are shown in Table 1. All manipulations with fish were carried out in compliance the Helsinki Declaration of the World Medical Association (2000) and the EEC Directive 86/609 EEC (1986) on the protection and welfare of experimental animals. Analysis of blood serum for total cholesterol (Ch), triglycerides, and cholesterol in fractions of very low density, low density, and high density lipoproteins (VLDL, LDL, and HDL) was performed in a Beckman Synchron CX4 multianalyzer (Beckman Coulter, United States) using corresponding diagnostic kits from Beckman Coulter (United States) as recommended by the manufacturer. The atherogenic coefficient (AC) was calculated by the equation: $A C=(C h$ $L D L+C h V L D L$ ) / Ch HDL (Martirosyan et al. 2007). Liver tissue fragments were fixed with $2 \%$ paraformaldehyde. The nuclei and lipid droplets in histological sections were stained with DAPI and Nile Red (Sigma-Aldrich, United States), respectively. Actin microfilaments were stained with FITC-conjugated phalloidin (Sigma-Aldrich, United States). The sections were scanned under an LSM-710 laser confocal microscope (Carl Zeiss, Germany), and their three-dimensional images were generated and processed using Imaris 7.2.3 software (Bitplane AG, Switzerland). Experimental data were processed by nonparametric statistical methods using the Statistica 10.0 program package. In particular, median values and interquartile ranges were calculated, and intergroup differences were evaluated by the Kruskal-Wallis test.

Table 1 - Morphometric features, weight and gender studied sculpins (Cottoidei) from Lake Baikal.

\begin{tabular}{lccccc}
\hline Species & n & Total length $(\mathbf{m m})$ & Weight $(\mathbf{g})$ & Gender & Age (years) \\
\hline Procottus major & 10 & $291 \pm 28$ & $114.5 \pm 23.1$ & + & $6 \pm 1.0$ \\
Cottocomephorus inermis & 10 & $148 \pm 10$ & $32.8 \pm 6.5$ & + & $3.5 \pm 0.5$ \\
Batrachocottus baicalensis & 10 & $147 \pm 6$ & $65.2 \pm 5.7$ & + & $6.5 \pm 0.5$ \\
Limnocottus bergianus & 10 & $222 \pm 12$ & $148.9 \pm 38.6$ & + & $6 \pm 1.0$ \\
\hline
\end{tabular}

The total blood cholesterol level in the studied sculpins proved to be species-specific (Fig. 1A). The lowest level was recorded in the pelagic species $C$. inermis $(143 \mathrm{mg} / \mathrm{dL})$. In general, the level recorded in this species is comparable to that in humans (Flaim et al. 1981). The values of this parameter in near-bottom species ( $P$. major, $L$. bergianus, and $B$. baicalensis) were higher (324.74 $-556.7 \mathrm{mg} / \mathrm{dL})$, being intermediate to total blood cholesterol concentrations determined in other fish species (Babin and Vernier 1989). A comparative analysis of the fractional composition of blood cholesterol revealed more profound differences between the sculpin species. The concentration of the antiatherogenic HDL fraction was the highest in P. major $(309.28 \mathrm{mg} / \mathrm{dL})$ and the lowest in C. inermis $(59.92 \mathrm{mg} / \mathrm{dL})$, Figure 1B. The concentration of LDL Ch was relatively low in $C$. inermis and $P$. major but high in $L$. bergianus and B. baicalensis (Fig. 1C). The concentration of VLDL Ch varied from 13.53 $\mathrm{mg} / \mathrm{dLin}$ C. inermis to $81.19 \mathrm{mg} / \mathrm{dL}$ in $P$. major (Fig. 1D). The observed features of blood lipid spectra account for interspecific differences in the antiatherogenic potential of blood (Fig. 1E, F). In $P$. major, antiatherogenic HDL Ch prevails over proatherogenic LDL and VLDL fractions, while the situation with $C$. inermis, B. baicalensis, and $L$. bergianus is inverse. Comparison of natural diets of Cottoidei species from Lake Baikal (Sideleva 2003) has shown that differences in blood lipid spectra between these fishes are not accounted for by their specific feeding habits and food spectra. Thus, although both $L$. bergianus and $P$. major feed mainly on amphipods, their blood lipid spectra drastically differ in the ratio of proatherogenic and antiatherogenic lipoprotein fractions. It is also noteworthy that the diet of $B$. baicalensis, the most active predator among the Baikal Cottoidei, presents very high atherogenic risk (70\% of amphipods and 30\% of other sculpin species), but its blood lipid spectrum is most 
similar to that of L. bergianus, which feeds mainly on amphipods. This is conclusive evidence that the relative contents of blood lipoprotein fractions in these closely related species are determined genetically.
A
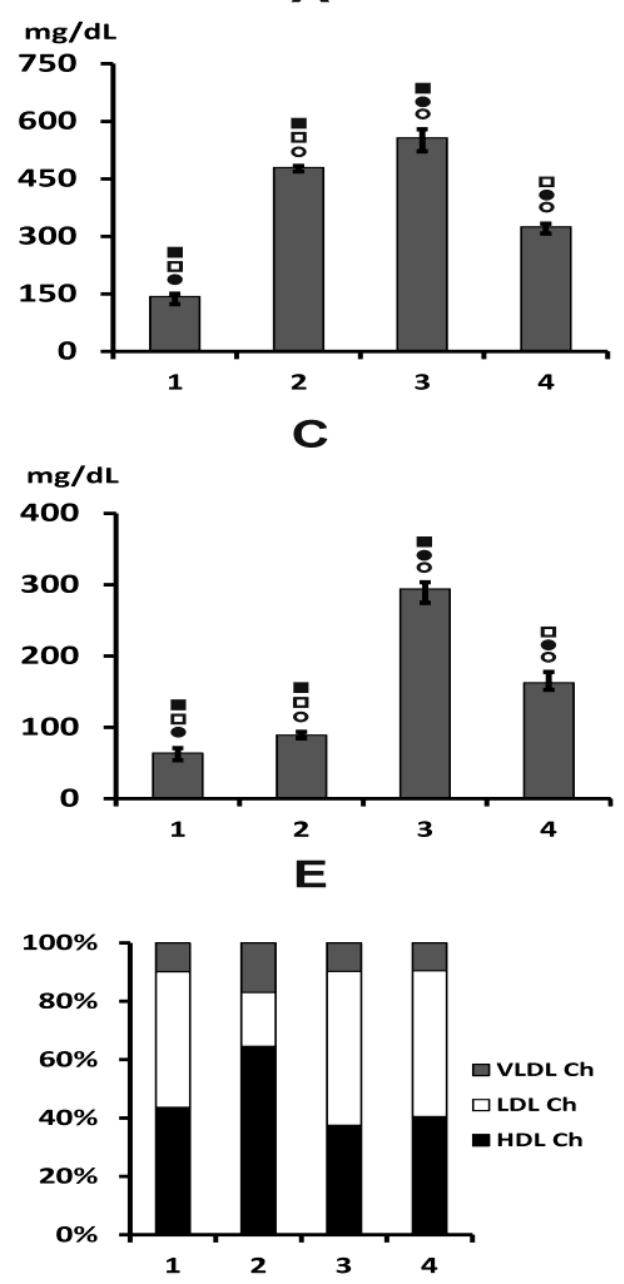

B
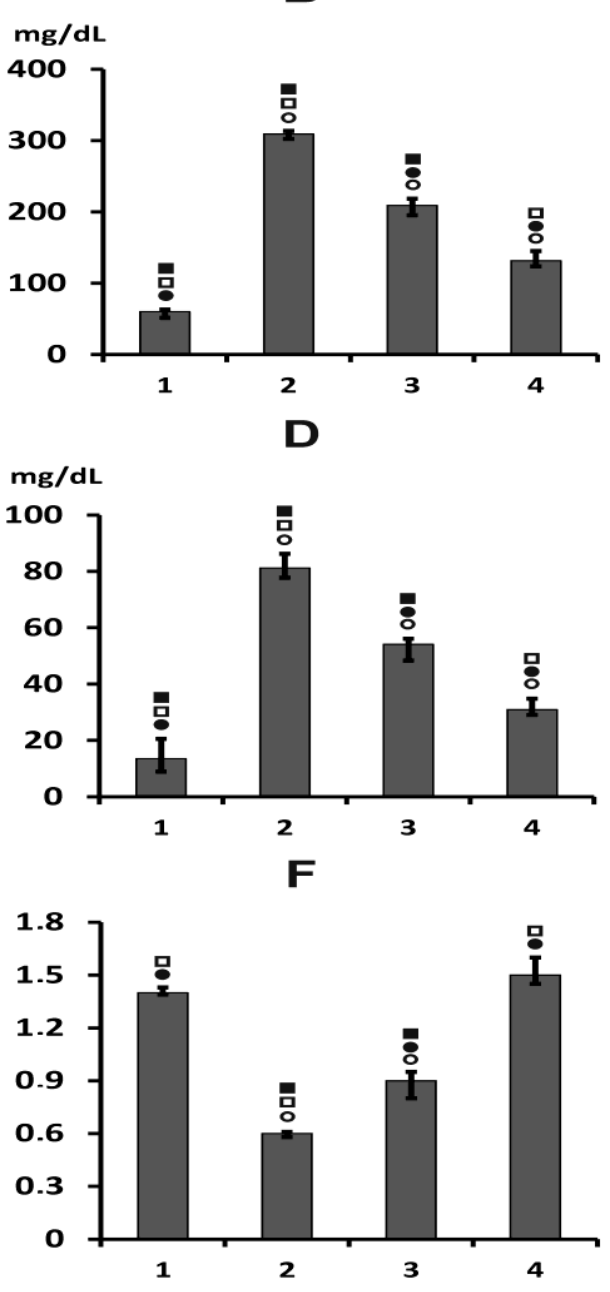

Figure 1 - Concentrations of (A) total cholesterol (Ch), (B) HDL Ch, (C) LDL Ch, (D) VLDL Ch, (E) ratios of blood cholesterol fractions, and $(\mathrm{F})$ coefficients of their atherogenicity in the blood of Baikal sculpins (median values and interquartile ranges). (1) Cottocomephorus inermis, (2) Procottus major, (3) Limnocottus bergianus, and (4) Batrachocottus baicalensis. Intergroup differences are evaluated by the Kruskal-Wallis test: $\circ p \geq 0.05$ compared to $C$. inermis; $\bullet p \geq 0.05$ compared to $P$. major; $\square p \geq 0.05$ compared to $L$. bergianus; $p \geq 0.05$ compared to B. baicalensis.

Analysis of Z-stacks of laser confocal microscopic images of preparations stained with lipid-specific Nile Red fluorescent dye has shown that the liver of Baikal sculpins contains numerous cells with large lipid droplets (Fig. 2). This is in agreement with the results of biochemical analysis of liver tissue in some Cottoidei, which confirm the high content of lipids in this organ ( $\mathrm{Ju}$ et al. 1997; Kozlova 1998). Therefore, these droplets in Baikal sculpins are an essential component of normal liver cells, rather than pathological structures such as those observed in mammals with steatosis (Takahashi et al. 2012).

Thus, the closely related Cottoidei species endemic to Lake Baikal have distinct speciesspecific features of blood lipid spectrum. Despite the common origin of these species, their representatives are characterized by a fairly wide concentration range of total blood cholesterol and its fractions. Studies on mechanisms responsible 
for these interspecific differences can provide an insight into the general principles of cholesterol metabolism. Since Baikal sculpins are characterized by prevalence of either antiatherogenic or proatherogenic lipoproteins, it appears expedient to separate this into corresponding groups and perform their comparative analysis. Of special interest is that the normal liver of Baikal sculpins can accumulate large amounts of lipids. This fact offers prospects for the study of natural mechanisms counteracting the deleterious effect of significant lipid deposits of the liver cells as well as on the maintenance of antiatherogenic potential in the whole body. All these data characterize the Baikal sculpins as a unique set of model organisms for the study of genetic, physiological, and ecological mechanisms of resistance to dyslipidemia and atherosclerosis.

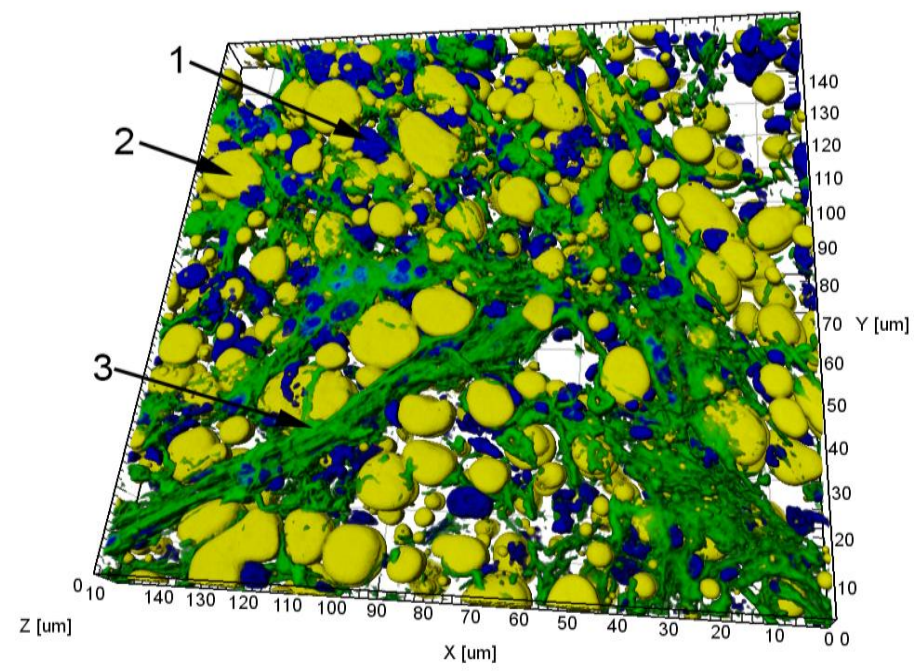

Figure 2 - Numerous large and small lipid droplets in the liver cells of $P$. major. Cell nuclei are stained with DAPI (1); lipid droplets, with Nile Red (2); and actin microfilaments, with FITC-conjugated phalloidin (3).

\section{ABBREVIATIONS}

Ch, cholesterol; HDL, high density lipoproteins; LDL, low density lipoproteins; VLDL, very low density lipoproteins.

\section{REFERENCES}

Babin PJ, Vernier JM. Plasma lipoproteins in fish. $J$ Lipid Res. 1989; 30: 467-489.

Getz GS, Reardon CA. Animal models of atherosclerosis. Arterioscler Thromb Vasc Biol. 2012; 32: 1104-1115.

Flaim E, Ferreri LF, Thye FW, Hill JE, Ritchey SJ. Plasma lipid and lipoprotein cholesterol concentrations in adult males consuming normal and high cholesterol diets under controlled conditions. Amer J Clin Nutr. 1981; 34: 1103-1108.

Henderson RJ, Torcher DR. The lipid composition and biochemistry of freshwater fish. Prog Lipid Res. 1987; 26: 281-347.
Ju S-J, Kucklick JR, Kozlova T, Harvey RH. Lipid accumulation and fatty acid composition during maturation of three pelagic fish species in Lake Baikal. J Great Lakes Res. 1997; 23: 241-253.

Kozlova TA. Lipid class composition of benthic-pelagic fishes (Cottocomephorus, Cottoidei) from Lake Baikal. Fish Physiol Biochem. 1998; 19: 211-216.

Martirosyan DM, Miroshnichenko LA, Kulokawa SN, Pogojeva AV, Zoloedov VI. Amaranth oil application for heart disease and hypertension. Lipids Health Dis. 2007; 6:1.

Shimaraev MN, Parfenova VV, Kostornova TYa, Domysheva VM, Gnatovskii RYu, Zhdanov AA, et al. Exchange processes and distribution of microorganisms in the deep zone of Lake Baikal. Dokl Biol Sci. 2000; 372: 279-282.

Sideleva VG. Endemic fishes of Lake Baikal. Leiden. Netherlands: Backhuys Publishers; 2003.

Takahashi Y, Soejima Y, Fukusato T. Animal models of nonalcoholic fatty liver disease/nonalcoholic steatohepatitis. World J Gastroenterol. 2012; 18: 2300-2308. 